\title{
Rheological Behaviors of Polyacrylonitrile/1-Butyl-3- Methylimidazolium Chloride Concentrated Solutions
}

\author{
Weiwei Liu, Lingyan Cheng, Hongyan Zhang, Yumei Zhang, Huaping Wang * and Mingfang Yu \\ 1 State Key Laboratory for Modification of Fibers Materials, Donghua University, Shanghai 200051, \\ People's Republic of China
}

* Author to whom correspondence should be addressed. E-Mail: towardsun@mail.dhu.edu.cn

Received: 30 August 2006; in revised form: 8 February 2007 / Accepted: 12 February 2007 /

Published: 7 March 2007

\begin{abstract}
One of the room temperature ionic liquids (RTILs), 1-butyl-3-methylimidazolium chloride $([\mathrm{BMIM}] \mathrm{Cl})$ was chosen to prepare the concentrated solutions of Polyacrylonitrile (PAN). The rheological behaviors of the solutions were measured with rotational rheometry under different conditions, including temperatures, concentration, and molecular weight of PAN. The solutions exhibited shear-thinning behaviors, similar to that of PAN/DMF solutions. The viscosities decreased with the increasing of shear rates. However, the viscosity decreased sharply at high shear rates when the concentration was up to $16 \mathrm{wt} \%$. The dependence of the viscosity on temperature was analyzed through the determination of the apparent activation energy. Unusually, the viscosity of solutions of higher concentration is lower than that of lower concentration. Similarly, the viscosity of low molecular weight PAN was higher than high molecular weight PAN at high shear rates. The dynamic rheological measurement indicates the loss modulus is much higher than storage modulus. The trend of complex viscosity is similar with the result of static rheological measurement. The interaction between PAN and ionic liquid [BMIM]Cl was discussed.
\end{abstract}

Keywords: Polyacrylonitrile; Ionic liquids; Rheological behaviours.

\section{Introduction}

Polyacrylonitrile (PAN) are commercially important and used for the precursor for highperformance carbon fiber. In the fiber applications, PAN is dissolved in a suitable solvent and spin to 
fibers by a wet or dry spinning process ${ }^{[1]}$. Several solvents have been used to prepare PAN spinning solutions. The extensively used solvents are aqueous sodium sulfocyanate (NaSCN), N, Ndimethylformamide (DMF), N, N-dimethylacetamide (DMAc), and N, N-dimethyl sulfone (DMSO). Nitric acid and aqueous zinc chloride $\left(\mathrm{ZnCl}_{2}\right)$ also can be used. Unfortunately, all the solvents mentioned above are unfriendly to the environment.

To meet the requirements of 'Green Chemistry', scientists have been searching for a green solvent to be an alternative to these solvents. In recent years, room temperature ionic liquids (RTILs) have received a lot of attention as the potential "green" and "designable" solvents ${ }^{[2]}$. Although the ionic liquids have been studied since the $1950 \mathrm{~s}{ }^{[3]}$, but they can not to be handled under an inert atmosphere. These are not part of our studies until some kinds of water-stable and air-stable ionic liquids are found such as $[\mathrm{BMIM}] \mathrm{Cl}$. Because of its extremely low volatility, some of the RTILs are promising environment-friendly solvents instead of the volatile organic solvents in a range of science and technology applications such as media for organic and inorganic reactions ${ }^{[4,5]}$, materials processing ${ }^{[6]}$ electrochemistry ${ }^{[7]}$ and chemical separation ${ }^{[8]}$.

During the copolymerization of AN and MMA in RTILs our research group find that RTILs are good solvents for PAN copolymer, and PAN copolymer could be precipitated from the RTILs solutions by the addition of water. Therefore RTILs are promising to be green solvents for PAN. Understanding the rheological properties of a solution is a convenient and effective way to gain a fundamental knowledge of the spinnability and structure-property relationships for the spinning solution. Thus, this paper investigates the rheological properties of PAN copolymer/BMIMCl concentrated solutions in some detail.

\section{Experimental}

\subsection{Materials}

The PAN copolymer, chlorobutane, 1-methylimidazole, ethyl acetate and acetone were used as supplied. The $\mathrm{P}(\mathrm{AN}-\mathrm{co}-\mathrm{MMA})$ copolymer ratio was 90:10. $\mathrm{P}(\mathrm{AN}-\mathrm{co}-\mathrm{MMA})$ copolymer was referred to as PAN. [BMIM]Cl was prepared based on the reported procedures ${ }^{[9]}$.

\subsection{Preparation of concentrated solutions}

Firstly, PAN powder was swollen by [BMIM]Cl at room temperature to give a white, little viscous pulp. Secondly the pulp was heated to $90^{\circ} \mathrm{C}$ until a homogeneous, transparent solution was formed. The PAN/[BMIM]Cl solution of $5 \mathrm{wt} \%$ to $22 \mathrm{wt} \%$ concentration was obtained within 5 hours at $90^{\circ} \mathrm{C}$. Figure 1 shows the process of the dissolution of PAN with ionic liquids [BMIM] $\mathrm{Cl}$ at $90^{\circ} \mathrm{C}$ at the concentration of $10 \mathrm{wt} \%$. 


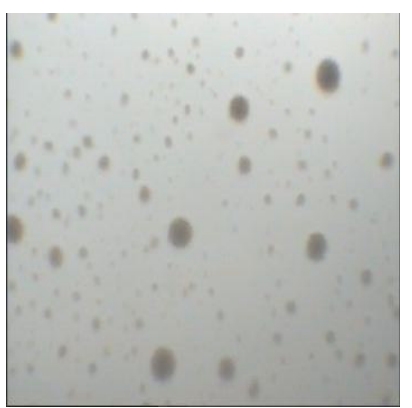

(a)

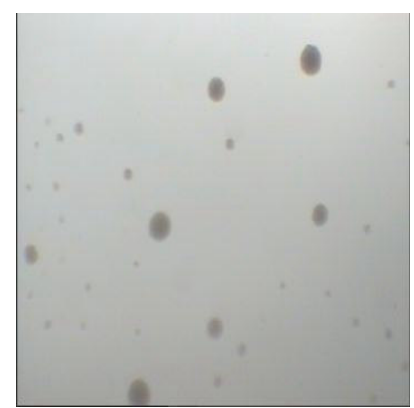

(b)

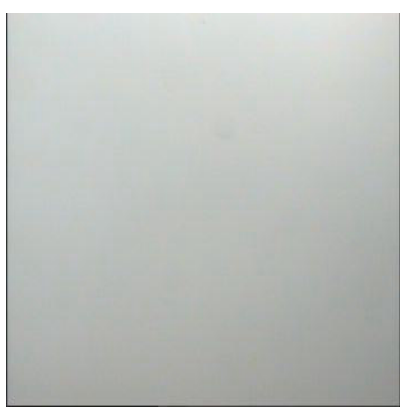

(c)

Figure 1. The Microscope image of PAN (pulp) dissolution in [BMIM]Cl at different time: (a) 20min, (b) $60 \mathrm{~min},(\mathrm{c}) 120 \mathrm{~min}$.

\subsection{Rheometry}

Steady state rheological measurements were performed on a HAAKE RS150L rotational rheometer using a $35 \mathrm{~mm}, 1^{\circ}$ cone and plate. The shear rate was linearly increased from 0.01 to $10001 / \mathrm{s}$ without oscillation. Dynamic rheological measurements were performed on a TA ARES-RFS rotational rheometer.

\subsection{Results and discussion}

The apparent viscosity $\eta_{a}$ is shown as a function of shear rate for the PAN in two different solvents, [BMIM]Cl and DMF (see Figure 2).

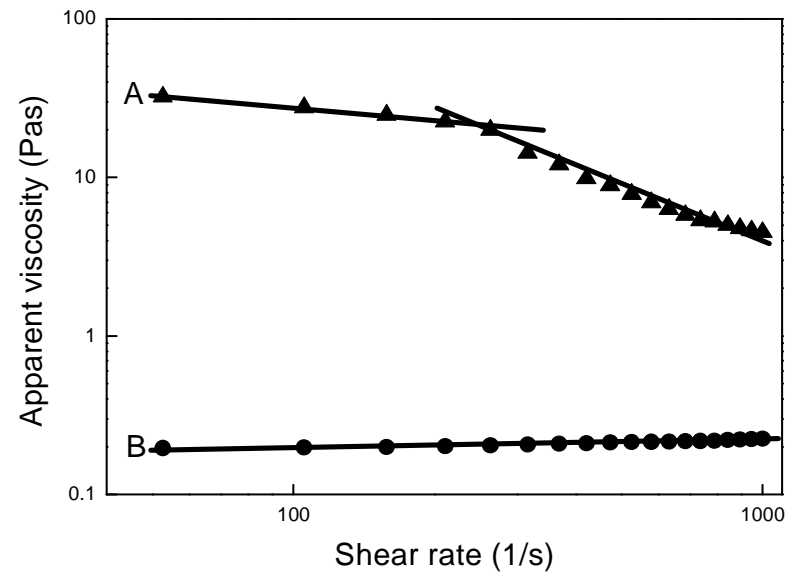

Figure 2. Rheological behavior of PAN $\left(14 \mathrm{wt} \%, 70^{\circ} \mathrm{C}\right)$ in the two solvents: (A) [BMIM]Cl; (B) DMF

Over the experimental range, the PAN/DMF solution is Newtonian fluid, while the PAN/[BMIM]Cl solution is pseudoplastic, that is, the apparent viscosity decreases with increasing shear rate. According to the so-called power law equation,

$$
\tau=K \dot{\gamma}^{n} \text { or } \eta_{a}=K \dot{\gamma}^{n-1}
$$


where $\mathrm{K}$ and $\mathrm{n}$ are constants, for pseudoplastic liquids the viscosity should decrease nearly linearly with shear rate on the log-log plot and the value of $n$ is less than one. However, it is surprising to find that the data of PAN/[BMIM]Cl solution should be considered having two linear stages (see Figure 2). The values of $\mathrm{n}$ and $\mathrm{R}^{2}$ are listed in Table 1 .

Table 1. The values of $\mathrm{n}$ for $\mathrm{PAN} /[\mathrm{BMIM}] \mathrm{Cl}$ solutions. $\mathrm{R}^{2}=$ statistical correlation coefficient.

\begin{tabular}{ccc}
\hline PAN/[BMIM]Cl & $\mathrm{n}$ & $\mathrm{R}^{2}$ \\
\hline Stage1 & 0.74504 & 0.993 \\
\hline Stage2 & -0.08613 & 0.981 \\
\hline
\end{tabular}

The value of $\mathrm{n}$ of the first stage is less than 1, according with the shear thinning rheological behavior. While at the second stage the apparent viscosity decreases so dramatically that even the value of $\mathrm{n}$ is negative. It is known that $\mathrm{n}$ is used to indicate the non-Newtonian property of fluids. The more the value of $n$ deviates from 1 , the more non-Newtonian the fluids would be. Usually, the value of $n$ of PAN/traditional organic solvents solutions is between 0 and 1 . Therefore, it could be postulated that there would be some much stronger interaction between PAN and the new solvent [BMIM]Cl which will be discussed in the following text.

The data in Figure 3 shows that the viscosity-shear rate curves for the PAN/[BMIM]Cl solutions of different concentrations.

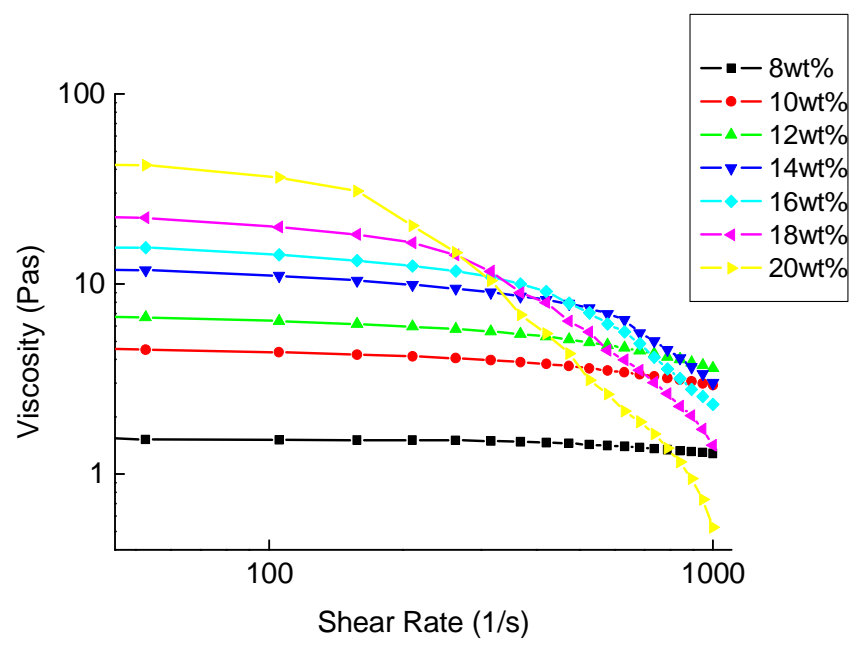

Figure 3. Rheological behavior of PAN/[BMIM]Cl solutions from $8 \mathrm{wt} \%$ to $22 \mathrm{wt} \%$ at $70^{\circ} \mathrm{C}$

These curves show that solutions of lower concentration remain Newtonian in behavior at high shear rates than concentrated solutions. At high concentration, the rheological behavior of $\mathrm{PAN} /[\mathrm{BMIM}] \mathrm{Cl}$ solutions acts like that of liquid crystalline polymers ${ }^{[10]}$ (LCP). The solvent $[\mathrm{BMIM}] \mathrm{Cl}$ reduces the number of entanglements. Reducing the number of entanglements at a given shear rate reduces the amount of orientation of macromolecular. Since the orientation of macromolecular is the major cause of non-Newtonian behavior, increasing the shear rate would make the non-Newtonian behavior more noticeable. Besides, it could be found in Figure 3 that when at high 
shear rate, the viscosity of high concentration solutions is lower than that of low concentration, for example, the viscosity of $22 \mathrm{wt} \%$ solution is the lowest among all the solutions when the shear rate is close to 1000 1/s. As we known, liquid crystalline polymers usually have rigid chain segment such as aromatic polyamide and aromatic polyester. The flexibility of the $\mathrm{C}-\mathrm{C}$ bond of the PAN main chain is smaller than the $\mathrm{C}-\mathrm{O}$ bond, $\mathrm{C}-\mathrm{N}$ bond and decrease because of the strong polarity of the $-\mathrm{CN}$, but comparing to the liquid crystalline polymers, the PAN chain segment can not be oriented because of lack of strong rigid chain segment. In this case, high concentration means more entanglements, which indicates that only low shear rates would be needed to orient the macromolecules. And the amount of entanglements is so large that there is no enough time and much more difficulties for most of them to slip and disengage. With the increasing of the shear rate the number of the oriented segments increases, so that the viscosity of the high concentration PAN/[BMIM]Cl solution decreases greatly.

The viscosity of most polymer solutions changes greatly with temperature. Commonly, the viscosity follows the Andrade or Arrhenius equation to a good approximation:

$$
\eta=A \exp \left(\frac{E}{R T}\right)
$$

In this equation, $\mathrm{A}$ is a constant, $\mathrm{E}$ is the activation energy. The slope of the straight line plotted by $\ln \eta$ versus $1 / \mathrm{T}$ is $\mathrm{E} / \mathrm{R}$. The value of the energy of activation depends strongly on whether the viscosities at various temperatures are evaluated at constant shear stress or at constant shear rate. Here $\mathrm{E}$ is evaluated at a constant shear rate. Figure 4 shows the viscosity as a function of temperature.

Here the two molecular weights are high enough to become entangled. Since low molecular weight polymers has fewer entanglements than high molecular weight ones, it is not surprising that deviations from Newtonian behavior start at higher shear rates for the low molecular weight solutions. However, in Figure 5, when the shear rate up to $210 \mathrm{1} / \mathrm{s}$ the viscosity of the high molecular weight $\mathrm{PAN} /[\mathrm{BMIM}] \mathrm{Cl}$ solution is lower than the other one.

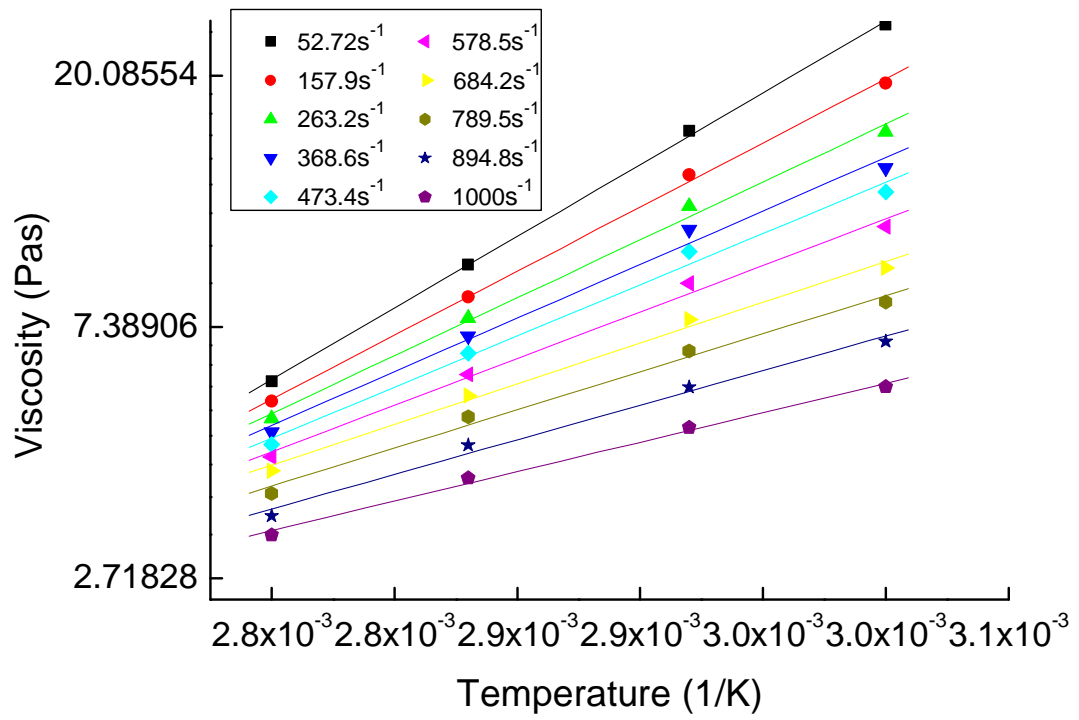

Figure 4. Viscosity as a function of temperature for $\mathrm{PAN} /[\mathrm{BMIM}] \mathrm{Cl}$ solution $(12 \mathrm{wt} \%)$ at different shear rates $(1 / \mathrm{s})$. 


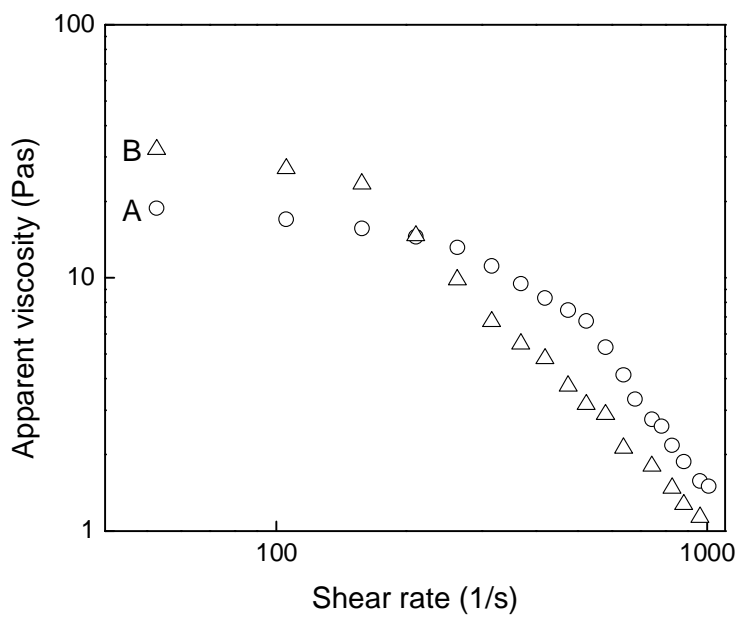

Figure 5. Rheological behavior of PAN/BMIM-Cl solutions $\left(90^{\circ} \mathrm{C}, 18 \mathrm{wt} \%\right)$ : $(\mathrm{A}) \mathrm{M}_{\eta}=45000$;

(B) $\mathrm{M}_{\eta}=60000$

The viscoelasticity of polymer is usually characterized by dynamic rheological experiment in which the sample is subjected to a sinusoidal strain of infinitesimal amplitude and fixed angular frequency. The term G', called the storage modulus, is the in-phase component of the modulus and represents the energy stored and recovered per cycle. Correspondingly, the term G", called the loss modulus, is the out-of-phase component of the modulus and represents the energy dissipated as heat per cycle of deformation. Figure 6 shows the modulus-angular frequency curves for the PAN/[BMIM]Cl solutions of different concentrations $\left(90^{\circ} \mathrm{C}\right)(\mathrm{a})$ and temperature $(14 \mathrm{wt} \%)(\mathrm{b})$. The data shows that the loss modulus G' is much higher than the storage modulus G'. Either module increases when the concentration increases or the temperature decreases. The storage modulus $G^{\prime}$ increases faster than the loss modulus G" because the elasticity of the solution increases.

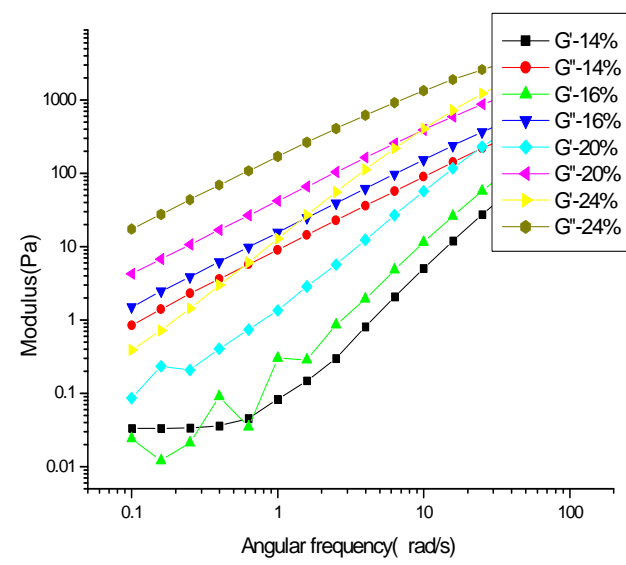

(a)

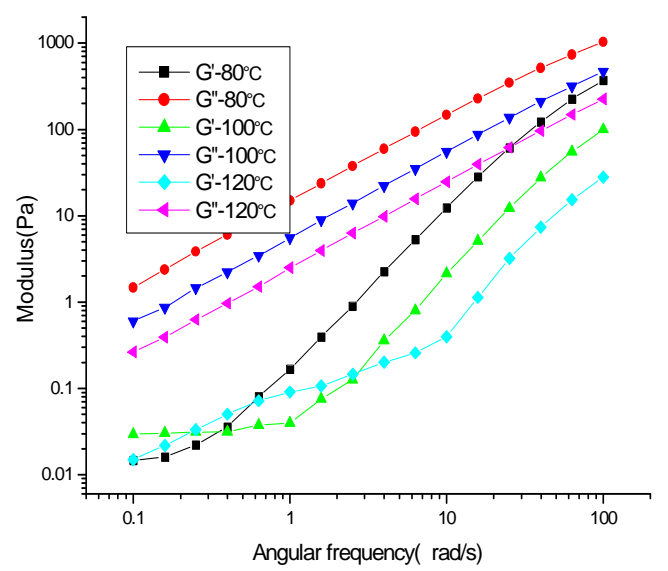

(b)

Figure 6. The modulus-angular frequency curves for the PAN/[BMIM]Cl solutions of different concentrations $\left(90^{\circ} \mathrm{C}\right)$ (a) and temperature $(14 \mathrm{wt} \%)(\mathrm{b})$. 
The data in Fig. 7 shows that the complex viscosity-angular frequency curves for the $\mathrm{PAN} /[\mathrm{BMIM}] \mathrm{Cl}$ solutions of different concentrations $\left(90^{\circ} \mathrm{C}\right)(\mathrm{a})$ and temperature $(14 \mathrm{wt} \%)(\mathrm{b})$. The curves show that the complex viscosity decreases when the angular frequency increases. That means the complex viscosity also have pseudoplastic behavior. The complex viscosity of the solution increases when the concentration increases or the temperature decreases. The trend of complex viscosity is similar to the result of static rheological measurement. This non-Newtonian behavior is of tremendous practical importance in the processing and fabrication of polymer material. First, the decreased viscosity makes the polymer solution easier to process or squirt through small channels, such as spinning. Second, the decrease in viscosity is associated with the development of elasticity in the polymer solution. This elasticity produces such phenomena as die swell, or the "puff-up" of extruded strands. Molecular orientation in molded objects also is closely related to polymer elasticity.

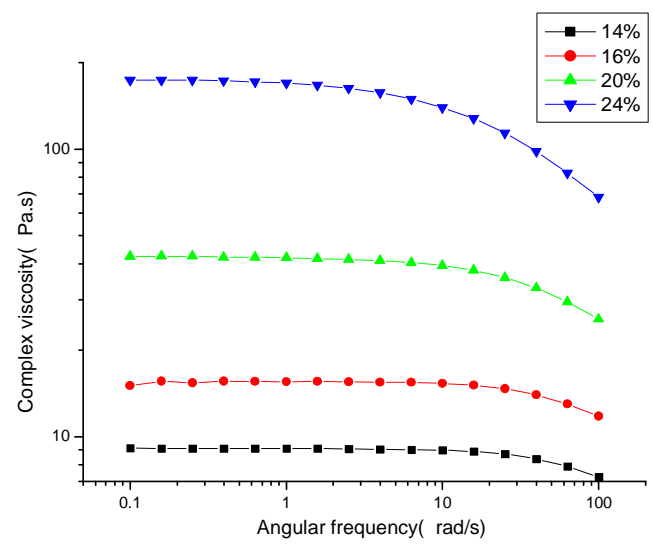

(a)

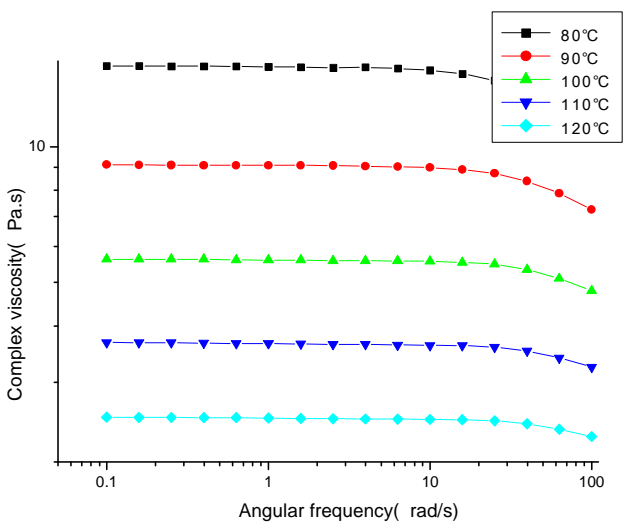

(b)

Figure 7. The complex viscosity-angular frequency curves for the PAN/[BMIM]Cl solutions of different concentrations $\left(90^{\circ} \mathrm{C}\right)(\mathrm{a})$ and temperature $(14 \mathrm{wt} \%)(\mathrm{b})$.

The possible dissolution mechanism of PAN in ionic liquid [BMIM]Cl is shown in Figure 8. It has been proved that the miscibility of ionic liquid with polymer is the function of their structural characteristic $^{[11]}$. We here focus on the polar group of PAN which has strong interaction with the ionic liquid. Because the electronegativity of the nitrogen atom on the nitrile grouping is very strong when the electron density is very large, the nitrogen atom on the nitrile grouping donate electrons and the carbon atom on the nitrile grouping attract electrons. As a result, there is strong interaction between the cation and the nitrogen atom on the nitrile grouping. Similarly, there is strong interaction between the anion and the carbon atom on the nitrile grouping. Similar interaction was also identified by other researchers $^{[12]}$.

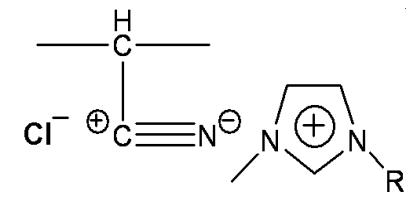

Figure 8. The interaction between PAN and [BMIM]Cl. 


\section{Conclusions}

Within the limits of the experimental techniques accessible to us, the results obtained for PAN/[BMIM]Cl solutions show that they are pseudoplastic, similar to the PAN/traditional organic solvents solutions. The viscosity decreases with the increasing of shear rate. The dependence on concentration and molecular weight shows that entanglements play an important role in the rheological behaviors of PAN/[BMIM]Cl concentrated solutions. The $\mathrm{E}_{\eta}$ decrease when the shear rates increase. The dynamic rheological measurement indicates the elasticity of the solution increases when the concentration is increasing or the temperature decreasing. The trend of complex viscosity is similar with the result of static rheological measurement. The interaction between PAN and ionic liquid $[\mathrm{BMIM}] \mathrm{Cl}$ is discussed. The rheological behaviors of the concentrated solution at high share rate are directive to the spinning of PAN fiber.

\section{Acknowledgements}

The authors would like to thank Ministry of Education of the People's Republic of China (105078), Science and Technology Commission of Shanghai Municipality (04JC14011) and Shanghai Municipal Education Commission (DAWN Project) for their financial support.

\section{References and Notes}

1. Minagawa, M.; Takasu, T.; Morita, T.; Shirai, H.; Fujikura, Y.; Kameda, Y. The steric effect of solvent molecules in the dissolution of polyacrylonitrile from five different N,Ndimethylformamide derivatives as studied using Raman spectroscopy. Polymer 1996, 37, 463-467.

2. Rogers, R.D.; Sedden, K.R. Ionic Liquids-Solvents of the Future. Science, 2003, 302, 792-793.

3. Hurley, F.H. and Wier, T.P. Electrodeposition of Metals from Fused Quaternary Ammonium Salts, J. Electrochem. SOC. 1951, 98, 203-206.

4. Welton, T. Room-Temperature Ionic Liquids. Solvents for Synthesis and Catalysis. Chem. Rev. 1999, 99, 2071-2082.

5. Chiappe, C.; Pieraccini, D. Ionic Liquids: Solvent Properties and Organic Reactivity J .Phys .Org. Chem. 2005, 18, 275-297.

6. Kubisa, P. Application of Ionic Liquids as Solvents for Polymerization Processes. Prog. Polym. Sci. 2004, 29, 3-12.

7. Carlin, R.T.; Osteryoung, R.A.; Wilkes, J.S. and Rovang, J. Studies of Titanium(IV) Chloride in a Strongly Lewis Acidic Molten Salt: Electrochemistry and Titanium NMR and Electronic Spectroscopy. Inorg. Chem. 1990, 29, 3003-3009.

8. Huddleston, J.G.; Willauer, H.D.; Swatloski, R.P.; Visser, A.E.; Rogers, R.D. Room Temperature Ionic Liquids as Novel Media for 'Clean' Liquid-Liquid Extraction. Chem. Commun. 1998, 16, 1765-1766.

9. Najdanovic-Visak, V.; Esperanca, J.M.; Rebelo, L.P.; Nunes da Ponte, M.; Guedes, H.J.; Seddon, K.R.; Szydlowski, J. Phase Behaviour of Room Temperature Ionic Liquid Solutions: An Unusually Large Co-Solvent Effect in (Water + Ethanol). Phys. Chem. Chem. Phys. 2002, 4, 1701-1703. 
10. Tanaka, H.; Gomez, M.A.; Tonelli, A.E.; Chichester-Hicks, S.V.; Haddon, R.C.; Bell, T.; Bell, A.T. Studies of the phase transitions in poly[bis(4-ethylphenoxy)phosphazene] and poly[bis(4tertbutylphenoxy)phosphazene]. 1. Phosphorus-31 NMR study. Macromolecules, 1989, 22(3), 1031-1036.

11. Marwanta, E.; Mizumo, T.; Nakamura, N.; Ohno, H. Improved ionic conductivity of nitrile rubber/ionic liquid composites. Polymer 2005, 46, 3795-3800.

12. Ferry, A.; Edman, L.; Forsyth, M.; MacFarlane, D.R.; Sun, J. NMR and Raman studies of a novel fast-ion-conducting polymer-in-salt electrolyte based on $\mathrm{LiCF}_{3} \mathrm{SO}_{3}$ and PAN Electrochim. Acta 2000, 45, 1237-1242.

(C) 2007 by MDPI (http://www.mdpi.org). Reproduction is permitted for noncommercial purposes. 